\title{
Participation of wind power producers in day-ahead and balancing markets: An overview and a simulation-based study
}

\author{
Hugo Algarvio ${ }^{1,2}$ | Fernando Lopes ${ }^{1} \mid$ António Couto ${ }^{1} \mid$ Ana Estanqueiro ${ }^{1}$
}

${ }^{1}$ LNEG-National Research Institute, Lisbon, Portugal

${ }^{2}$ Instituto Superior Técnico, Universidade de Lisboa, Lisbon, Portugal

Correspondence

Hugo Algarvio, UER - Renewable Energy and Energy Systems Integration Unit, LNEG, Lisbon, Portugal.

Email: hugo.algarvio@tecnico.ulisboa.pt

Funding information

FCT (Fundação para a Ciência e Tecnologia), Grant/Award Number: PD/BD/105863/2014
At present, a harmonized pan-European electricity market (EM) is a close reality. While in day-ahead markets (DAMs) the harmonization is at an advanced stage, in balancing markets (BMs) still exist some challenging issues, notably the remuneration of imbalances: some countries have simple and clear methods, but others consider complex methods that are not appealing to the participation of variable renewable energy (VRE). The participation of VRE in BMs is technically feasible, although with some restrictions to guarantee security and stability. Thus the economic attractiveness of these markets should be increased in order to enable full integration of VRE without feed-in-tariffs or other incentives. This article presents an overview of EMs, focusing on European BMs, and also investigates the benefits of the participation of wind power producers (WPPs) in BMs at both economic and technical levels. In particular, the article presents a new strategy allowing WPPs to bid in BMs. It also presents a study involving four scenarios, where WPPs participate in: (a) the DAM (baseline scenario), (b) the DAM and the automatic-activated frequency restoration reserve market, (c) the DAM and the manually activated FRR (mFRR) market, and (d) the DAM and a 15-min mFRR market. The simulations are performed with the agent-based system MATREM (for Multi-Agent TRading in EMs). For the last scenario, the results indicate an increase around 6\% in the wind energy value to the market, a decrease of $12 \%$ in the total reserve used, and a decrease around $16 \%$ in the costs from the BM.

This article is categorized under:

Wind Power $>$ Systems and Infrastructure

Wind Power $>$ Economics and Policy

Energy Systems Analysis > Economics and Policy

\section{KEYWORDS}

agent-based system, balancing markets, simulation-based study, strategic bidding, wind power producers

\section{I INTRODUCTION}

The share of variable renewable energy (VRE) in power supply systems is progressively increasing, while the share of conventional generation is decreasing. To ensure that the power supply continue functioning reliably and in a cost-effective way, electricity markets (EMs) should be adapted to this new paradigm (e.g., Lopes \& Coelho, 2018a). Contrary to conventional generation, appropriately designed to control the power output, VRE units are nondispatchable and dependent of stochastic weather conditions. Since these conditions can be forecasted, the possible contributions from VRE can be considered schedulable, although with uncertainties associated with forecast errors. In order to minimize such uncertainties, the input of VRE should be scheduled as close as possible to real-time operation. 